SCIENTIFIC REPORT

\title{
Reduced response of retinal vessel diameters to flicker stimulation in patients with diabetes
}

\author{
G Garhöfer, C Zawinka, H Resch, P Kothy, L Schmetterer, G T Dorner
}

Br J Ophthalmol 2004;88:887-891. doi: 10.1136/bjo.2003.033548

Background/aim: Stimulation of the retina with flickering light increases retinal arterial and venous diameters in animals and humans, indicating a tight coupling between neural activity and blood flow. The aim of the present study was to investigate whether this response is altered in patients with insulin dependent diabetes mellitus.

Methods: 26 patients with diabetes mellitus with no or mild non-proliferative retinopathy and 26 age and sex matched healthy volunteers were included in the study. Retinal vessel diameters were measured continuously with the Zeiss retinal vessel analyser. During these measurements three episodes of square wave flicker stimulation periods $(16,32$, and 64 seconds; $8 \mathrm{~Hz}$ ) were applied through the illumination pathway of the vessel analyser.

Results: In retinal arteries, the response to stimulation with diffuse luminance flicker was significantly diminished in diabetic patients compared to healthy volunteers (ANOVA, $\mathrm{p}<0.0031)$. In non-diabetic controls flicker stimulation increased retinal arterial diameters by $+1.6 \%(1.8 \%)$ (mean, $p<0.001 v$ baseline), $+2.8 \%$ (SD 2.2\%) $(p<0.001)$ and $+2.8 \%(1.6 \%)(p<0.001)$ during 16,32 , and 64 seconds of flicker stimulation, respectively. In diabetic patients flicker had no effect on arterial vessel diameters: $+0.1 \%(3.1 \%)$ (16 seconds, $p=0.9),+1.1 \%(2.7 \%)$ (32 seconds, $p=0.07$ ), $+1.0 \%(2.8 \%)(64$ seconds, $p=0.1)$. In retinal veins, the response to flicker light was not significantly different in both groups. Retinal venous vessel diameters increased by $+0.7 \%$ (1.6\%) (16 seconds, $\mathrm{p}<0.05),+1.9 \%(2.3 \%)$ (32 seconds, $p<0.001)$ and $1.7 \%(1.8 \%)(64$ seconds, $p<0.001)$ in controls during flicker stimulation. Again, no increase was observed in the patients group: $+0.6 \%(2.4 \%),+0.5 \%(1.5 \%)$, and $+1.2 \%(3.1 \%)(16,32$, and 64 seconds, respectively). Conclusion: Flicker responses of retinal arteries and veins are abnormally reduced in patients with IDDM with no or mild non-proliferative retinopathy. Whether this diminished response can be attributed to altered retinal vascular reactivity or to decreased neural activity has yet to be clarified.

$\mathrm{T}$ he retina has the ability to regulate blood flow in response to different metabolic demands. Several animal and human studies demonstrated an increase in retinal vessel diameters ${ }^{1-3}$ as well as in retinal ${ }^{4}$ and optic nerve head blood flow $^{5-8}$ during stimulation with diffuse luminance flicker. Like in the brain, blood flow in retinal vessels is strongly coupled to neural activity, which was recently confirmed using laser Doppler flowmetry ${ }^{5}$ and retinal vessel diameter measurements. ${ }^{129}$

Little information, however, is available about this regulatory process in the diseased retina. A variety of studies indicate that ocular blood flow is altered in diabetes and retinal perfusion abnormalities have been proposed to contribute to the pathogenesis of diabetic retinopathy. ${ }^{10}{ }^{11}$ It is even speculated that the impairment of blood flow regulation in the retina precedes the earliest visible signs of diabetic retinal complications. ${ }^{12}$ Several studies indicate abnormal retinal blood flow regulation in patients with diabetes. This has been shown for the vasoconstrictor response to hyperoxia ${ }^{13}{ }^{14}$ as well as for the autoregulatory response of the retina. ${ }^{15} 16$

Thus, it was the aim of the present study to determine whether the vasomotor response of retinal vessels to increased neuronal activity is altered in patients with early stage insulin dependent diabetes mellitus. For this purpose, the Zeiss retinal vessel analyser, a commercially available tool for real time determination of retinal vessel diameters has been adapted to allow for determination of retinal vessel diameters during flickering light stimulation.

\section{METHODS}

\section{Subjects}

The study protocol was approved by the ethics committee of the Vienna University School of Medicine and followed the guidelines set forth in the Declaration of Helsinki. All patients signed a written informed consent and passed a screening examination before the study day.

Twenty six patients with early IDDM and 26 control subjects matched for sex and age, body mass index between 15th and 85th percentile were enrolled in this assessor masked, controlled clinical study. Every subject had to pass an ophthalmic examination including slit lamp biomicroscopy, indirect funduscopy, and measurement of intraocular pressure (IOP) with Goldmann applanation tonometry. Inclusion criteria of patients were insulin dependent diabetes mellitus (IDDM) with non or mild non-proliferative retinopathy. The eyes were classified according to the Modified Airlie House Classification. ${ }^{17}{ }^{18}$ Patients with no signs of diabetic retinopathy (level 1) or patients with one or more microaneurysms (level 2) were included. In all subjects the right eye was studied.

Exclusion criteria were non-insulin dependent diabetes, maturity onset diabetes of the young (MODY diabetes), any sign of non-diabetes induced vascular pathologies, systemic hypertension (defined as systolic blood pressure $>150 \mathrm{~mm} \mathrm{Hg}$, diastolic blood pressure $>90 \mathrm{~mm} \mathrm{Hg}$ ), treatment with vasoactive drugs except insulin, presence of intraocular pathology other than diabetic retinopathy, ametropia of less than 3 dioptres, and anisometropia of less than 1 dioptre.

\section{Zeiss retinal vessel analyser (RVA)}

Retinal vessel diameters were evaluated with the RVA. The RVA is a commercially available system which comprises a fundus camera (Zeiss FF 450, Jena, Germany), a video camera, a high resolution video recorder, a real time monitor, and a personal computer with a vessel diameter analysing 
Table 1 Main patient characteristics

\begin{tabular}{|c|c|c|}
\hline & Diabetes group & Control group \\
\hline Sex (male/female) & $14 / 12$ & $14 / 12$ \\
\hline Mean age (years) & $47(14)$ & $47(14)$ \\
\hline $\mathrm{HbA}_{\mathrm{lc}}(\%)$ & $6.6(1.0)$ & - \\
\hline Diabetes duration (years) & $10(8)$ & - \\
\hline Intraocular pressure (mm Hg) & $13(2)$ & $13(2)$ \\
\hline MAP $(\mathrm{mm} \mathrm{Hg})$ & $83(10)$ & $86(11)$ \\
\hline Diabetic retinopathy (level 1/level 2$)^{*}$ & $12 / 14)$ & \\
\hline
\end{tabular}

software. The RVA allows the precise determination of retinal vessels' diameter with a time resolution of 25 readings/ second. The fundus was illuminated with light in the range of wavelengths between 567 and $587 \mathrm{~nm}$. In this spectral range, the contrast between retinal vessels and the surrounding tissue is optimal. Retinal irradiance was approximately $220 \mu \mathrm{W} \mathrm{cm}{ }^{-2}$, which is approximately 50 times lower than the maximum level allowed for constant illumination of the retina at the wavelengths mentioned above. The system provides excellent reproducibility and sensitivity. ${ }^{19}$ In the present study, a major temporal artery and vein was studied. Measurements of retinal vessel diameters were taken between 1 and 2 disc diameters from the margin of the optic disc.

\section{Flickering light stimulation}

For flicker stimulation a custom built device was used, stimulating with light flashes at a frequency of $8 \mathrm{~Hz}$. Flicker was generated by focusing the light of a $150 \mathrm{~W}$ halogen light source on a rotating sector disc producing a square wave light pattern with a modulation depth of $100 \%$. Using an optical fibre, flicker stimuli were delivered to the eye through the illumination pathways of the fundus camera of the RVA. The flicker was centred in the macula with an angle of approximately 30 degrees.

A wavelength separation technique was used to spectrally separate the flicker light from that used to illuminate the fundus. For flicker stimulation, white light in combination with a $550 \mathrm{~nm}$ low pass cut-off filter was used. This ensures that only light with wavelengths below $550 \mathrm{~nm}$ is used for flicker stimulation. To separate the flickering light from the light illuminating the fundus, an interference filter with a centre wavelength of $577 \mathrm{~nm}$ and a bandwidth of $10 \mathrm{~nm}$ (Laser Components, Olchingen, Germany) was placed in front of the light source of the fundus camera. This window was chosen, because in this spectral range, the contrast between blood vessels and the surrounding tissue is optimal. A retinal irradiance of approximately $220 \mu \mathrm{W} \mathrm{cm}{ }^{-2}$ was used to achieve optimal fundus images. A second interference filter, which exactly matches the one in the illumination pathway is placed in front of the video camera. This ensures that the light used for flicker stimulation does not reach the CCD chip of the video camera, but is perceived by the subject under study.

\section{Assessment of $\mathrm{HbA}_{1 \mathrm{c}}$ values}

Blood $\mathrm{HbA}_{1 \mathrm{c}}$ values were assessed using a standard HPLC laboratory method by the Department of Clinical Chemistry, University of Vienna, Austria.

\section{Experimental paradigm}

All measurements were done in the right eye according to the following time schedule. After a short resting period to obtain stable haemodynamic conditions, diameter measurements with the RVA were performed. Retinal vessel diameters were continuously measured for 352 seconds. Diffuse luminance flickering light was applied consecutively for 16, 32, and 64 seconds at a frequency of $8 \mathrm{~Hz}$ during measurements with the RVA. Before and after each flicker period 60 seconds of baseline recordings were recorded.

\section{Statistics}

Changes in retinal vessel diameters were expressed as percentage change over baseline values. Baseline values were calculated as an average of the last 30 seconds before start of the flicker stimulation. Flicker response of the 16 seconds flicker period was calculated as an average of the last 10 seconds of the stimulation period, for the 32 second stimulation period as an average of the last 20 seconds of the stimulation period and for the 64 seconds stimulation as an average of the last 30 seconds of the visual stimulation. Repeated measures ANOVA was used for statistical analysis according to the following model: 2 (diabetes/control) $\times 3$ (flicker duration: 16/32/64 s) $\times 2$ (experiment: baseline/ flicker:). Because of three steps repeated measurements in one factor (flicker duration of 16/32/64 seconds), a multivariate approach was used in order to preserve sphericity. Post hoc analysis was performed using two tailed paired $t$ tests. To ensure that the overall chance of making a type I error is still less than 0.05, Bonferroni correction for multiple testing was applied. Shapiro-Wilks' W test was used to test for normal data distribution. Calculations were performed using the Statistica software package (Statsoft, USA). Data are presented as means (SD).

\section{RESULTS}

Because of insufficient target fixation, two measurements of retinal arteries and two measurements of veins of diabetic
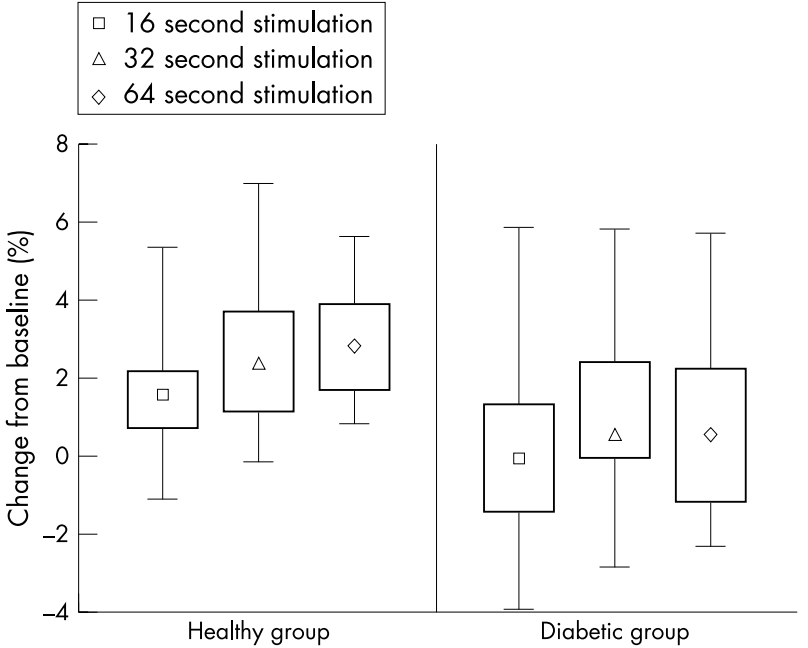

Figure 1 Box (mean, 25\% and 75\% Cl) and whisker (5\% and 95\% Cl) plot of flicker response (percentage changes) as recorded after 16, 32, and 64 seconds of flicker stimulation. 
Table 2 Absolute arterial and venous vessel diameter before and during flicker stimulation. Data are presented as means (SD)

\begin{tabular}{|c|c|c|c|c|c|}
\hline & \multirow[b]{2}{*}{ Flicker time (seconds) } & \multicolumn{2}{|l|}{ Diabetes group } & \multicolumn{2}{|l|}{ Control group } \\
\hline & & Before flicker & During flicker & Before flicker & During flicker \\
\hline \multirow[t]{3}{*}{ Arterial vessel diameter $(\mu \mathrm{m})$} & 16 & $122.6(15.7)$ & $122.6(15.5)$ & $122.6(17.4)$ & $124.5(17.9)^{*}$ \\
\hline & 32 & $121.8(14.8)$ & $123.1(15.3)$ & $123.7(17.1)$ & $126.9(16.6)^{*}$ \\
\hline & 64 & $123.2(15.8)$ & $124.3(16.6)$ & 122.7 (18.3) & $126.2(18.8)^{*}$ \\
\hline \multirow[t]{3}{*}{ Venous vessel diameter $(\mu \mathrm{m})$} & 16 & $154.6(22.0)$ & $155.5(23.0)$ & $151.7(21.8)$ & $152.8(22.5)^{*}$ \\
\hline & 32 & $154.4(20.0)$ & $155.0(19.8)$ & $150.0(21.9)$ & $152.8(22.6)^{*}$ \\
\hline & 64 & $153.1(18.8)$ & $155.2(20.0)$ & $150.6(21.7)$ & $153.2(22.6)^{*}$ \\
\hline
\end{tabular}

patients had to be excluded from the analysis. The remaining data of 24 arteries and 24 veins have been used for data analysis. Main patients characteristics are given in table 1. Retinal vessel diameters, mean arterial pressure and intraocular pressure were comparable between both groups. Both retinal arterial and venous vessel diameters at the measurement site were comparable between patients with diabetes and healthy controls. Absolute values of retinal vessel diameters are given in table 2 .

In retinal arteries, the response to stimulation with diffuse luminance flicker was significantly diminished in diabetic patients compared to healthy volunteers (ANOVA, $\mathrm{p}=0.0031, \mathrm{df}=46, \mathrm{~F}=9.743$ ). In healthy controls, flicker stimulation increased retinal arterial vessel diameters by $+1.6 \% \quad(1.8 \%) \quad(\mathrm{p}<0.001, t$ test flicker $v$ baseline $),+2.8 \%$ $(2.2 \%)(\mathrm{p}<0.001)$ and $+2.8 \%(1.6 \%)(\mathrm{p}<0.001)$ during 16,32 , and 64 seconds of flicker, respectively. In diabetic patients, no effect of flickering light on arterial vessel diameters was observed: $+0.1 \%(3.1 \%)$ ( 16 seconds, $\mathrm{p}=0.9),+1.1 \%(2.7 \%)$ (32 seconds, $\mathrm{p}=0.07$ ), and $+1.0 \% \quad(2.8 \%) \quad$ (64 seconds, $\mathrm{p}=0.1)($ fig 1$)$.

Response of retinal venous diameters was not significantly different in diabetic compared to healthy volunteers (ANOVA, $\mathrm{p}=0.186, \mathrm{df}=46, \mathrm{~F}=1.804$ ). However, in diabetic patients no significant increase in venous vessel diameters was observed during stimulation with diffuse luminance flicker: +0.6 (2.4) (16 seconds, $\mathrm{p}<0.2$ ), +0.5 (1.5) (32 seconds, $\mathrm{p}<0.1)$, and +1.2 (3.1) (64 seconds, $\mathrm{p}<0.06)$. In contrast, retinal venous vessel diameters increased by $+0.7 \%$ ( $1.6 \%) \quad(16$ seconds, $\mathrm{p}<0.05)$, $+1.9 \% \quad(2.3 \%) \quad$ (32 seconds, $\mathrm{p}<0.001), 1.7 \%(1.8 \%) \quad(64$ seconds, $\mathrm{p}<0.001)$ in healthy volunteers during flicker stimulation.

No correlation was found between flicker response and $\mathrm{HbA}_{\mathrm{lc}}$ values or diabetes duration (data not shown).

\section{DISCUSSION}

The results of this study indicate that the response of retinal vessel diameters to diffuse luminance flicker is significantly reduced in patients with early stage diabetic retinopathy. Whereas retinal arteries and veins dilate in the order of $2-3 \%$ in healthy volunteers, no significant increase could be observed in patients with early stage diabetes.

There is compelling evidence now that vascular tone and blood flow regulation are affected in diabetic patients. Several studies revealed an increase in blood flow, induced by short time hyperglycaemia, mainly attributable to an increase in blood flow velocity. ${ }^{20-23}$ Furthermore, it has been shown that retinal perfusion is already increased in patients with early IDDM with a diabetes duration of less than 4 years $^{12}$ and that retinal vasodilatation may precede other signs of retinopathy. ${ }^{24}$ In contrast, other reports indicate that blood flow may be decreased in early stage diabetes..$^{25} 26$

In addition, the regulatory capacity of retinal vessels appears to be affected in the diseased retina. This has been shown in several animal and human studies showing that diabetes is associated with an abnormal retinal vascular response to hyperoxia ${ }^{13}{ }^{14}$ and abnormal retinal autoregulation. $^{15}{ }^{16}$ These results are compatible with the data of the current study indicating that the response of retinal vessels to stimulation with diffuse luminance flicker is significantly reduced in diabetic patients.

At least two mechanisms may be attributable to the blunted flicker response in diabetic patients. Firstly, the blunted flicker response in diabetic patients could reflect retinal damage caused by vascular abnormalities. Among others, one of the earliest alterations in diabetic retinas have been found to include pericyte loss, ${ }^{27}$ which could alter responsiveness to locally generated mediators. It has been suggested that diabetic patients have either increased nitric oxide (NO) synthase activity or decreased vascular sensitivity to NO. ${ }^{11}$ This is important for the results of the present study because NO appears to play a part in flicker induced vasodilatation..$^{328}$

On the other hand neural activity in the retina may be altered before any clinical signs of retinopathy are evident. Measurements of oscillatory potentials or pattern responses demonstrated that ERG abnormalities occur before any signs of clinically evident retinopathy. ${ }^{29}{ }^{30}$ Several lines of evidence indicate that the increase in retinal and optic nerve head blood flow in response to flicker stimulation may be a direct consequence of an increase in retinal neural activity, mainly attributable to increased ganglion cell activity. ${ }^{5}$ The increase in optic nerve head blood flow shows similar characteristics to the first and second harmonic amplitudes of the flicker electroretinogram, indicating that blood flow changes in response to flickering light stimulation are a consequence of increased neural activity. ${ }^{5}$ Hence the results of the present study may also be compatible with the idea of reduced neural activity and leading to a decreased flicker response in diabetes.

One has to consider that our results are limited by the fact that we can only provide information about retinal vessels diameter and the reactivity of retinal vessels to flicker stimulation, not about changes in blood flow per se. In view of the fact that blood flow in a main retinal vessel is $\pi \mathrm{D}^{2} \mathrm{~V}_{\text {mean }} / 4{ }^{31}$ where $\mathrm{D}$ is the vessel diameter and $\mathrm{V}$ the mean velocity of the blood, a twofold change in diameter produces a fourfold change in blood flow. Whereas this underlines the importance of getting information about vessel diameters for estimating blood flow in the retina, one has to keep in mind that for determination of blood flow, information on blood flow velocity is crucial. Thus, we cannot exclude that even though the diameters of the large retinal arteries and veins showed no measurable changes during flicker stimulation in the patients with diabetes, the blood flow may have increased at these sites owing to an increase in blood speed.

To finally answer this question, combined measurements of vessel diameters and red blood cell velocity, which would allow for determination of blood flow, would be necessary. In principle, bidirectional laser Doppler velocimetry is capable of measuring red blood velocity in major retinal vessel. ${ }^{32}$ However, to obtain reproducible flicker responses with this 
system is difficult, especially in patients, because excellent target fixation is required.

Interestingly, flicker response is altered in retinal arteries but failed to reach a level of significance in retinal veins. Whether this is simply related to the smaller percentage change in retinal veins during flicker stimulation is unclear. One has, however, to consider that vasodilatation of retinal veins is primarily a passive effect. As the microcirculation is the most important site of vascular resistance, data on the retinal microvasculature are required to elucidate this behaviour. Owing to the limited resolution of the RVA, we cannot finally answer this question. This problem applies also to the other methods that have been previously proposed to measure retinal vessel size in vivo. ${ }^{33-36}$

In the current study we measured the response of one retinal artery and vein only. Thus, we cannot exclude that retinal vessels were already predilated in diabetic patients, which could also contribute to the diminished flicker response. This is strengthened by the fact that the total retinal venous diameter, calculated by adding the cross section of each visible vein observed around the optic disc, is increased in patients with diabetes. ${ }^{12}$ However, changes in retinal vessel diameters between diabetics and healthy controls are difficult to evaluate because of the high interindividual differences in retinal vascular architecture.

In conclusion, retinal vasodilatation caused by stimulation with diffuse luminance flicker appears to be diminished in diabetic patients with no or mild diabetic retinopathy. This strengthens the hypothesis that retinal vascular dysregulation is an early process in diabetic retinopathy.

\section{ACKNOWLEDGEMENTS}

Financial support of the "Fonds zur Förderung der wissenschaftlichen Forschung" Grant No. P14262 is gratefully acknowledged. Furthermore, the authors thank Dr Selim Orgül, Basle for his advises in statistical analysis of the data.

\section{Authors' affiliations}

G Garhöfer, C Zawinka, H Resch, P Kothy, L Schmetterer, G T Dorner, Department of Clinical Pharmacology, University of Vienna, Austria G Garhöfer, G T Dorner, Department of Ophthalmology, University of Vienna, Austria

P Kothy, Department of Ophthalmology, Semmelweis University, Budapest, Hungary

L Schmetterer, Institute of Medical Physics, University of Vienna, Austria

Correspondence to: G T Dorner, Department of Clinical Pharmacology, Währinger Gürtel 18-20, A-1090 Vienna, Austria; guido.dorner@ univie.ac.at

Accepted for publication 1 December 2003

\section{REFERENCES}

1 Polak K, Schmetterer L, Riva CE. Influence of flicker frequency on flicker induced changes of retinal vessel diameters. Invest Ophthalmol Vis Sci 2002;43:2721-6

2 Dorner GT, Garhofer G, Huemer KH, et al. Hyperglycemia affects flickerinduced vasodilation in the retina of healthy subjects. Vis Res 2003:43:1495-500.

3 Dorner GT, Garhofer G, Kiss B, et al. Nitric oxide regulates retinal vascular tone in man. Am J Physiol Heart Circ Physiol 2003;285:631-6.

4 Michelson G, Patzelt A, Harazny J. Flickering light increases retinal blood flow. Retina 2002;22:336-43.

5 Falsini B, Riva CE, Logean E. Flicker-evoked changes in human optic nerve blood flow: relationship with retinal neural activity. Invest Ophthalmol Vis Sci 2002;43:2309-16.
6 Riva CE, Falsini B, Logean E. Flicker-evoked responses of human optic nerve head blood flow: luminance versus chromatic modulation. Invest Ophthalmo Vis Sci 2001;42:756-62

7 Buerk DG, Riva CE, Cranstoun SD. Nitric oxide has a vasodilatory role in cat optic nerve head during flicker stimuli. Microvasc Res 1996:52:13-26.

8 Buerk DG, Riva CE, Cranstoun SD. Frequency and luminance-dependent blood flow and $\mathrm{K}+$ ion changes during flicker stimuli in cat optic nerve head Invest Ophthalmol Vis Sci 1995;36:2216-27.

9 Formaz F, Riva C, Geiser M. Diffuse luminance flicker increases retinal vessel diameter in humans. Curr Eye Res 1997;16:1252-7.

10 Kohner EM. Patel V, Rassam SM. Role of blood flow and impaired autoregulation in the pathogenesis of diabetic retinopathy. Diabetes 1995; 44:603-7.

11 Schmetterer L, Wolzt M. Ocular blood flow and associated functiona deviations in diabetic retinopathy. Diabetologia 1999;42:387-405.

12 Grunwald JE, DuPont J, Riva CE. Retinal haemodynamics in patients with early diabetes mellitus. Br J Ophthalmol 1996;80:327-31.

13 Grunwald JE, Riva CE, Brucker AJ, et al. Altered retinal vascular response to $100 \%$ oxygen breathing in diabetes mellitus. Ophthalmology 1984;91:1447-52.

14 Patel V, Rassam SM, Chen HC, et al. Oxygen reactivity in diabetes mellitus: effect of hypertension and hyperglycaemia. Clin Sci (Lond) 1994;86:689-95.

15 Sinclair SH, Grunwald JE, Riva CE, et al. Retinal vascular autoregulation in diabetes mellitus. Ophthalmology 1982;89:748-50.

16 Rassam SM, Patel V, Kohner EM. The effect of experimental hypertension on retinal vascular autoregulation in humans: a mechanism for the progression of diabetic retinopathy. Exp Physiol 1995;80:53-68.

17 Diabetic Retinopathy Study. Report Number 6. Design, methods, and baseline results. Report Number 7. A modification of the Airlie House classification of diabetic retinopathy. Invest Ophthalmol Vis Sci 1981;21:1-226.

18 Early Treatment Diabetic Retinopathy Study Research Group. Grading diabetic retinopathy from stereoscopic color fundus photographs - an extension of the modified Airlie House classification. ETDRS report number 10. Ophthalmology 1991;98:786-806.

19 Polak K, Dorner GT, Kiss B, et al. Evaluation of the Zeiss retinal vessel analyser. Br J Ophthalmol 2000;84:1285-90.

20 Atherton A, Hill DW, Keen $\mathrm{H}$, et al. The effect of acute hyperglycaemia on the retinal circulation of the normal cat. Diabetologia 1980;18:233-7.

21 Ernest JT, Goldstick TK, Engerman RL. Hyperglycemia impairs retinal oxygen autoregulation in normal and diabetic dogs. Invest Ophthalmol Vis Sci 1983;24:985-9.

22 Sullivan PM, Davies GE, Caldwell G, et al. Retinal blood flow during hyperglycemia. A laser Doppler velocimetry study. Invest Ophthalmol Vis Sci 1990;31:2041-5.

23 Luksch A, Polak K, Matulla B, et al. Glucose and insulin exert additive ocula and renal vasodilator effects on healthy humans. Diabetologia 2001:44:95-103

24 Falck A, Laatikainen L. Retinal vasodilation and hyperglycaemia in diabetic children and adolescents. Acta Ophthalmol Scand 1995;73:119-24.

25 Arend O, Wolf S, Harris A, et al. The relationship of macular microcirculation to visual acuity in diabetic patients. Arch Ophthalmol 1995;113:610-4.

26 Bursell SE, Clermont AC, Kinsley BT, et al. Retinal blood flow changes in patients with insulin-dependent diabetes mellitus and no diabetic retinopathy. Invest Ophthalmol Vis Sci 1996;37:886-97.

27 Paget $C$, Lecomte $M$, Ruggiero $D$, et al. Modification of enzymatic antioxidants in retinal microvascular cells by glucose or advanced glycation end products. Free Radic Biol Med 1998;25:121-9.

28 Kondo M, Wang L, Bill A. The role of nitric oxide in hyperaemic response to flicker in the retina and optic nerve in cats. Acta Ophthalmol Scand 1997:75:232-5

29 Li Q, Zemel E, Miller B, et al. Early retinal damage in experimental diabetes: electroretinographical and morphological observations. Exp Eye Res 2002; 74:615-25

30 Juen S, Kieselbach GF. Electrophysiological changes in juvenile diabetics without retinopathy. Arch Ophthalmol 1990;108:372-5.

31 Ganong W. Review of medical physiology. New YorK: Lange Medical, 1995.

32 Riva CE, Grunwald JE, Sinclair SH, et al. Blood velocity and volumetric flow rate in human retinal vessels. Invest Ophthalmol Vis Sci 1985;26:1124-32.

33 Delori FC, Fitch KA, Feke GT, et al. Evaluation of micrometric and microdensitometric methods for measuring the width of retinal vessel images on fundus photographs. Graefes Arch Clin Exp Ophthalmol 1988;226:393-9

34 Chen H, Patel V, Wiek J, et al. Vessel diameter changes during the cardiac cycle. Eye 1994:8:97-103

35 Rassam SM, Patel V, Brinchmann-Hansen O, et al. Accurate vessel width measurement from fundus photographs: a new concept. Br J Ophthalmol 1994:78:24-9.

36 Suzuki Y. Direct measurement of retinal vessel diameter: comparison with microdensitometric methods based on fundus photographs. Surv Ophthalmol 1995;39(Suppl 1):S57-65. 


\section{$\mathrm{ECHO}$}

Treatment for amblyopia can wait until school entry

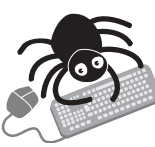

Please visit the British Journal of

Ophthalmology website [www. bjophthalmol. com] for a link to the full text of this article.
Treatment for mild amblyopia in one eye can safely be left beyond preschool years, according to a randomised controlled trial in the UK. The implications are far reaching as screening and treatment are practised world wide.

The single blind trial established that full treatment with glasses and eye patch benefited preschool children (age 3-5 years) with moderate (6/36-6/18) amblyopia, whose improved vision translated into one or two lines of the Snellen chart, but not those with mild (6/9-6/ 12) amblyopia over untreated children. Six months afterwards, when all children in need of glasses had them, the difference in visual acuity among the three groups was minimal, further suggesting that delay did no harm.

The trial assessed standard treatment for amblyopia—a patch and glasses-and glasses alone against no treatment in 177 children with amblyopia identified at standard preschool screening and referred to one of eight children's eye clinics. Impairment ranged from 6/9-6/ 36. Children randomised to treatment had an initial assessment and reassessments at 24, 52, 54, and 78 weeks. Those receiving a patch and glasses had their patch fitted after six weeks' wearing glasses, if indicated. Glasses were prescribed after 52 weeks for any child in any group who needed them and eye patches two weeks later.

Preschool screening is widespread owing to the belief that amblyopia is treatable only up to age 7 years, but its cost effectiveness and psychological impact have been queried. A systematic review has previously claimed that evidence in favour of treatment is lacking.

A British Medical Journal 2003;327:1251-1254. 